\title{
A search for the near-infrared counterpart of the eclipsing millisecond X-ray pulsar Swift J1749.4-2807^
}

\author{
P. D’Avanzo ${ }^{1}$, S. Campana ${ }^{1}$, T. Muñoz-Darias ${ }^{1}$, T. Belloni ${ }^{1}$, E. Bozzo ${ }^{2}$, M. Falanga ${ }^{3}$, and L. Stella ${ }^{4}$ \\ 1 INAF, Osservatorio Astronomico di Brera, via E. Bianchi 46, 23807 Merate (LC), Italy \\ e-mail: paolo.davanzo@brera.inaf.it \\ 2 ISDC Data Center for Astrophysics of the University of Geneva, chemin d'Écogia 16, 1290 Versoix, Switzerland \\ 3 International Space Science Institute (ISSI), Hallerstrasse 6, 3012 Bern, Switzerland \\ ${ }^{4}$ INAF, Osservatorio Astronomico di Roma, via Frascati 33, 00040 Monteporzio Catone, Italy
}

Received 5 August 2011 / Accepted 29 August 2011

\section{ABSTRACT}

\begin{abstract}
Context. Swift J1749.4-2807 is a transient accreting millisecond X-ray pulsars and the first to display X-ray eclipses. It therefore holds a strong potential for accurate mass measurements in a low-mass X-ray binary system.

Aims. Determining the companion star's radial velocity would make it possible to fully resolve the system and to accurately measure the mass of the neutron star based on dynamical measurements. Unfortunately, no optical or near infrared (NIR) counterpart has been identified to date for this system, whether in outburst or in quiescence.

Methods. We performed a photometric study of the field of Swift J1749.4-2807 during quiescence to search for a variable counterpart. The source direction lies on the Galactic plane, making any search for its optical/NIR counterpart challenging. To minimize the effects of field crowding and interstellar extinction, we carried out our observations using the adaptive optics NIR imager NACO mounted at the ESO Very Large Telescope (VLT).

Results. From the analysis of public Swift X-ray data obtained during outburst, we derived the most precise (1.6" radius) position for this source. Due to the extreme stellar crowding of the field, 41 sources are detected in our VLT images within the X-ray error circle, with some of them possibly showing variability consistent with the expectations.

Conclusions. We carried out the first deep imaging campaign devoted to the search for the quiescent NIR counterpart of Swift J1749.4-2807. Our results allow us to provide constraints on the nature of the companion star of this system. Furthermore, they suggest that future phase-resolved NIR observations (performed with large-aperture telescopes and adaptive optics) covering the full orbital period of the system are likely to identify the quiescent counterpart of Swift J1749.4-2807, through measuring its orbital variability, opening the possibility of dynamical studies of this unique source.
\end{abstract}

Key words. X-rays: binaries - binaries: eclipsing

\section{Introduction}

Accreting millisecond X-ray pulsars (AMXPs) are a subclass of low-mass X-ray binary transients that host an old, weakly magnetic $\left(10^{8}-10^{9} \mathrm{G}\right)$ neutron star that accretes mass from a companion star and has been spun up to millisecond periods via transfer of angular momentum (for a review see Wijnands 2006). Since 1998, fourteen such systems have been discovered, with orbital periods in the range of $40 \mathrm{~min}$ to $19 \mathrm{~h}$ and spin frequencies from 1.7 to $5.4 \mathrm{~ms}$ (Wijnands \& van der Klis 1998; Chakrabarty \& Morgan 1998; Markwardt et al. 2002; Galloway et al. 2002; Campana et al. 2003; Strohmayer et al. 2003; Galloway et al. 2005; Kaaret et al. 2006; Krimm et al. 2007; Casella et al. 2008; Altamirano et al. 2008; Altamirano et al. 2010; Papitto et al. 2010; Markwardt \& Strohmayer 2010; Altamirano et al. 2011; Papitto et al. 2011). Since they have been spun up by accretion (see, e.g., Falanga et al. 2005), their initial mass should have increased by at least $0.1-0.2 M_{\odot}$ compared to the canonical neutron star mass (e.g., Thorsett \& Chakrabarty 1999). AMXPs are therefore excellent systems for tightly constraining the neutron star equation of state, a key result for fundamental physics.

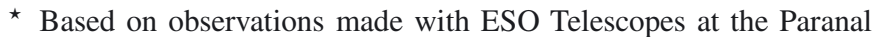
Observatory under programme ID 285.D-5030(A).
The most direct way to determine the mass of an object in a binary system is through dynamical measurements. These sources are ideal candidates for such studies because by measuring the delays in the arrival times of the pulsations it is possible to derive the system's orbital period and the radial velocity of the neutron star $\left(K_{1}\right)$ with high precision. In addition, during the quiescent phase of transient $\mathrm{X}$-ray binaries, the projected radial velocity of the companion star $\left(K_{2}\right)$ can be measured through phase-resolved optical spectroscopy, when it is possible to study the light from the companion star without a sizeable contamination from the accretion disc (see, e.g., Hynes 2010). Alternatively, the same measurements can be carried out during outburst by measuring the Doppler shifts of narrow emission lines in the Bowen blend, originating on the irradiated face of the companion star (Steeghs \& Casares 2002). The only missing ingredient to fully resolve the system is its inclination $(i)$, which is usually very hard to determine with precision. The presence of eclipses is very helpful in this respect (although these are observed only rarely).

The AMXP Swift J1749.4-2807 was discovered in June 2006 by the Swift satellite when a type I burst from it was observed, which was emitted during a dim outburst (Wijnands et al. 2009; Campana 2009). The transient resumed activity in April 2010. During this outburst, observations carried out with 


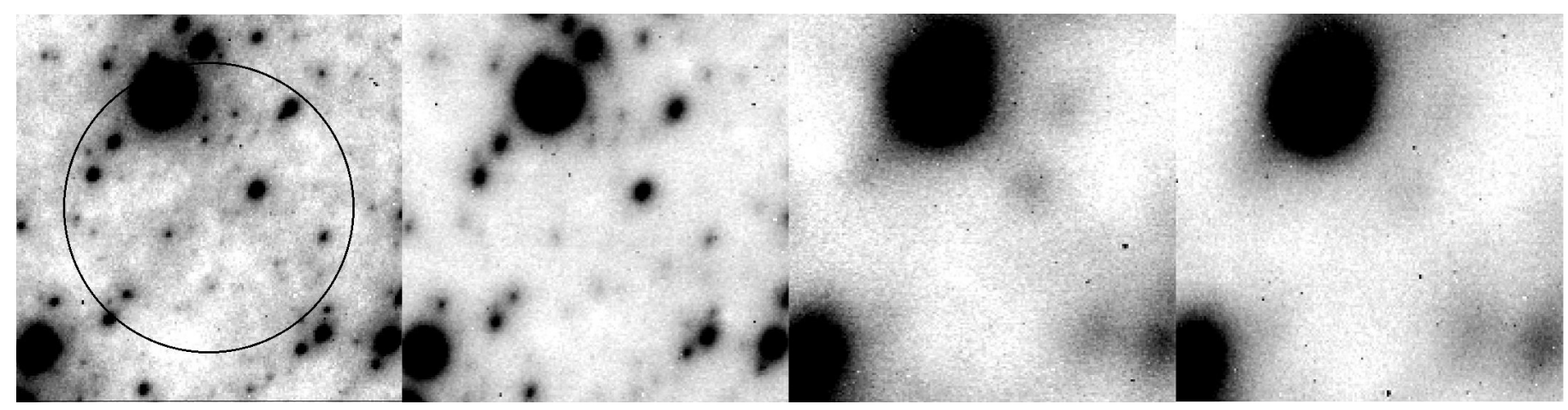

Fig. 1. From left to right. The field of Swift J1749.4-2807 observed in the $H$-band on 2010 Aug. 30, Aug. 31, Sept. 01, Sept. 05. A clear worsening of resolution and flux limit is visible in the last two epochs (see Sect. 2 for details). The black circle represents the X-ray error circle (1.6" radius, $90 \%$ c.1.). North is up and east to the left. Image size is $5.4^{\prime \prime} \times 5.4^{\prime \prime}$.

Table 1. Observation log.

\begin{tabular}{lcclcc}
\hline \hline $\begin{array}{l}\text { UT mid observation } \\
\text { (YYYmmdd) }\end{array}$ & $\begin{array}{c}\text { Exposure } \\
(\mathrm{s})\end{array}$ & $\begin{array}{c}\text { PSF } \\
(\operatorname{arcsec})\end{array}$ & Instrument & Filter & Orbital phase \\
\hline $20100830.0474(3)$ & $7 \times 2 \times 60 \mathrm{~s}$ & $0.10^{\prime \prime}$ & VLT/NACO & $H$ & $0.747(9)$ \\
$20100831.0533(3)$ & $7 \times 2 \times 60 \mathrm{~s}$ & $0.17^{\prime \prime}$ & VLT/NACO & $H$ & $0.485(9)$ \\
$20100901.0632(3)$ & $7 \times 2 \times 60 \mathrm{~s}$ & $0.62^{\prime \prime}$ & VLT/NACO & $H$ & $0.234(9)$ \\
$20100905.1124(3)$ & $7 \times 2 \times 60 \mathrm{~s}$ & $0.71^{\prime \prime}$ & VLT/NACO & $H$ & $0.256(9)$ \\
\hline
\end{tabular}

the Rossi X-ray Timing Explorer (RossiXTE) satellite led to the discovery of a neutron star spin period of $1.9 \mathrm{~ms}$ and of an orbital period of $8.82 \mathrm{~h}$ (Markwardt \& Strohmayer 2010; Altamirano et al. 2011). Short eclipses (with a duration of 2200 s) were observed in X-ray from observations with RossiXTE. In the RossiXTE and Swift-XRT light curves folded at the orbital period, marked flux decreases occur near orbital longitude 90 degrees (as measured from the ascending node), i.e. when the neutron star is expected to be behind the companion. RossiXTE observations cover two separate eclipse egresses and one ingress (Markwardt \& Strohmayer 2010). At least one X-ray eclipse was also observed in Swift-XRT data during the 2010 outburst (Ferrigno et al. 2011). During this eclipse, a residual flux is clearly seen in the Swift-XRT data. The flux reduces by a factor 3.3. This was ascribed to a dust-scattering halo located along the line of sight to Swift J1749.4-2807 (Ferrigno et al. 2011). $\mathrm{X}$-ray pulsations are not detected during these intervals, testifying to their genuine eclipse nature (Markwardt \& Strohmayer 2010; Altamirano et al. 2011; Ferrigno et al. 2011). The detection of X-ray eclipses enables the system inclination to be constrained in the $74.4^{\circ}-77.9^{\circ}$ range (depending on the assumed neutron star mass; Markwardt \& Strohmayer 2010; Altamirano et al. 2011).

Swift J1749.4-2807 is the only known AMXP showing eclipses, so it would be a very promising low mass X-ray binary system for dynamical studies aimed at precise mass measurements. As discussed above, by determining $K_{2}$, it will be possible to precisely determine the mass of the neutron star with dynamical measurements. To this end, it is mandatory to identify its optical or near infrared (NIR) counterpart. Unfortunately, the source lies in the Galactic plane, in the direction of an extremely crowded and exinct region, making any search for its optical/NIR counterpart challenging. During the 2006 and 2010 outbursts, no optical/NIR counterpart was detected (Kubanek et al. 2006; Khamitov et al. 2006; Blustin et al. 2006; Melandri et al. 2006; Yang et al. 2010). Furthermore, no X-ray counterpart could be detected with a $1.1 \mathrm{ks}$ observation carried out with the
Chandra satellite during the fading phase of the 2010 outburst (Chakrabarty et al. 2010).

We present here the results of the first observational campaign devoted to identifying the NIR quiescent counterpart of Swift J1749.4-2807. To minimize the effects of field crowding and interstellar extinction, we carried out our observations using the adaptive optics NIR imager NACO mounted at the ESO Very large Telescope (VLT).

\section{Observations and results}

We observed the field of Swift J1749.4-2807 with our approved DDT programme ID 285.D-5030(A) at the ESO VLT. Observations were carried out in service mode on 2010 Aug. 30, 31, Sept. 1 and 5 with NAos COnica (NACO), the adaptive optics (AO) NIR imager and spectrometer mounted at the VLT UT4 telescope. We used the S27 camera, which has a pixel size of $0.027^{\prime \prime}$ and a field of view of $28^{\prime \prime} \times 28^{\prime \prime}$. As a reference for the AO correction, we used the GSC-2 star S222131225301 $(V=14.7)$, located $18^{\prime \prime}$ away from our target. The visual dichroic element and wavefront sensor were used. The observation $\log$ is presented in Table 1 . Image reduction was carried out using the NACO pipeline data reduction, part of the ECLIPSE $^{1}$ package. The images were dark-subtracted, flat-field and bad and hot pixels corrected, and sky-subtracted. To increase the signal-to-noise ratio, we averaged the images taken on each night. Unfortunately, the last two epochs of observations (Sep. 1 and Sep. 5) were affected by natural seeing $>1^{\prime \prime}$, with a resulting poorer resolution and shallower flux limit with respect to the others so were not included in our analysis (Fig. 1). Therefore, all the analysis and results presented in the following refer to the observing epochs of Aug. 30 and Aug. 31. Astrometry was carried out by using the USNOB $1.0^{2}$ and the $2 \mathrm{MASS}^{3}$ catalogues as reference with a resulting accuracy of $0.2^{\prime \prime}$. Point spread function

\footnotetext{
${ }^{1}$ http://www.eso.org/projects/aot/eclipse/

2 http://www.nofs.navy.mil/data/fchpix/

3 http://www.ipac.caltech.edu/2mass/
} 
(PSF)-photometry was made with the ESO-MIDAS ${ }^{4}$ DAOPHOT task for all the objects in the field. The photometric calibration was done against standard stars. To minimize any systematic effect, we performed differential photometry with respect to a selection of local isolated and non-saturated reference stars.

We analysed the X-ray Swift-XRT data obtained during the 2006 and 2010 outbursts. We derived an enhanced position for the 2006 and 2010 outbursts, respectively, using the Leicester University interface ${ }^{5}$. We then combined the two enhanced positions to derive the following coordinates: $\operatorname{RA}(J 2000)=17: 49: 31.83$, Dec $(J 2000)=-28: 08: 04.7$ with an uncertainty radius of $1.6^{\prime \prime}$ ( $90 \%$ c.l.). This is the most precise position for this source obtained so far. As shown in Fig. 1, in spite of its relatively small size, the error circle is quite crowded, since the direction is located towards the Galactic plane. Future Chandra X-ray observations would significantly reduce the uncertainty in the source position.

The VLT observing epochs were chosen so as to have a phase difference of $0.25 P_{\text {orb }}$ (based on the precise ephemeris of Altamirano et al. 2011). On Aug. 30.05 UT the source was observed at phase $\sim 0.75$ (ascending node), while on Aug. 31.05 it was at phase $\sim 0.5$ (inferior conjunction, i.e. when the observer sees the side of the companion star that faces the compact object). Under the assumption that the companion star is subject to irradiation from the compact object, as observed in all the compact $\left(P_{\text {orb }}<4.3 \mathrm{~h}\right)$ AMXPs for which a well-sampled optical light curve was obtained (D'Avanzo et al. 2009 and references therein), an increase in luminosity of a few tenths of magnitude is expected between the two epochs, making identification of the NIR counterpart of the system easier. On the other hand, the $8.82 \mathrm{~h}$ orbital period of Swift J1749.4-2807 implies a larger orbital separation than that of compact AMXPs, possibly reducing the effects of irradiation. In this case, a "classical" ellipsoidal modulation is expected for the optical/NIR light curve (as observed for the $P_{\text {orb }} \sim 19$ h AMXP Aql X-1; Shahbaz et al. 1998; Welsh et al. 2000) resulting in a fading between the two epochs.

\subsection{Candidate NIR counterparts of Swift J1749.4-2807}

To verify the presence of variable candidates, we carried out PSF-photometry of all the objects visible (above $3 \sigma$ c.l.) in both images, which fall within the X-ray error circle. 41 sources satisfy these criteria. We plot in Fig. 2 the measured $H$-band magnitudes of epoch 1 vs. epoch 2 . As expected, the dispersion increases for fainter objects. Apart from four objects (marked as "ABCD") no source is seen to significantly $(\geq 3 \sigma)$ vary more than $0.09,0.27,0.44$, and 0.58 mag for objects with $H<21$, $21<H<22,22<H<23$, and $23<H<24$ mag, respectively. To investigate further the presence of any variable object, we performed image subtraction with the ISIS package (Alard 2000; Alard \& Lupton 1998) on our two images, using the image obtained on Aug. 30 as a reference. The result of the image subtraction analysis is shown in the right hand panel of Fig. 3. No significant residual is present within the X-ray error circle (excluding the regions close to the brightest star of the field). We note that the background varies considerably between the two epochs, possibly affecting the result of the image subtraction. It is thus possible that such background fluctuations overcome the scatter observed with PSF photometry in the subtracted image. This would imply either that source variability is hidden in the intrinsic dispersion of our photometry or that the

\footnotetext{
${ }^{4}$ http://www.eso.org/projects/esomidas/

5 http://www.swift.ac.uk/user_objects/
}

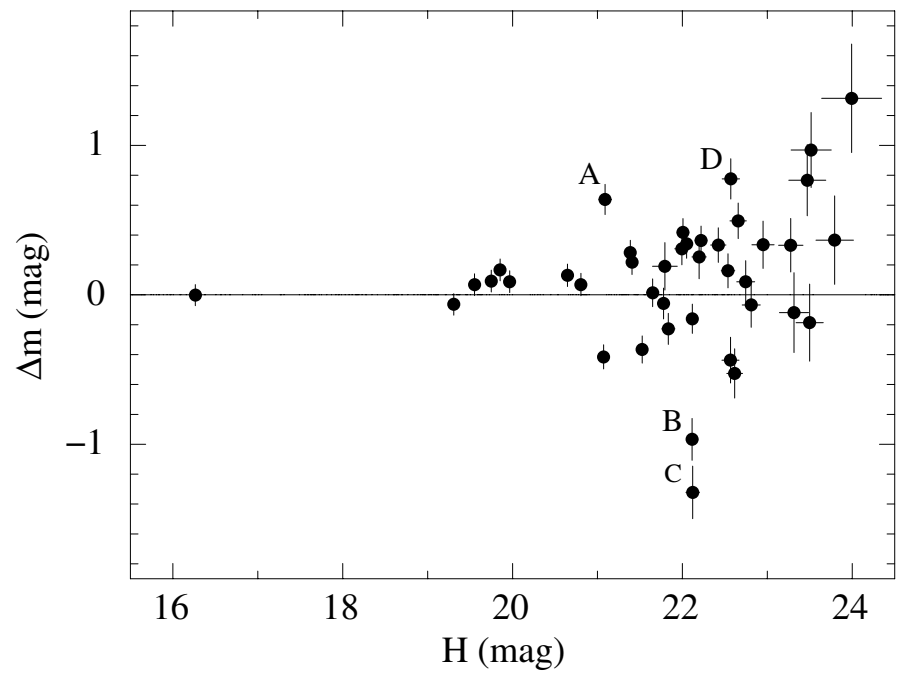

Fig. 2. $H$-band magnitude of sources detected within the X-ray error circle (measured on 2010 Aug. 30) vs. the magnitude difference between the Aug. 30 and Aug. 31 epochs. The objects with $\Delta m>0$ mag are brighter in the second epoch. Sources marked as "ABCD" show variability greater than the observed dispersion for objects with similar brightness. Errors are at $1 \sigma$ c.l., magnitudes are in the Vega system and uncorrected for Galactic extinction.

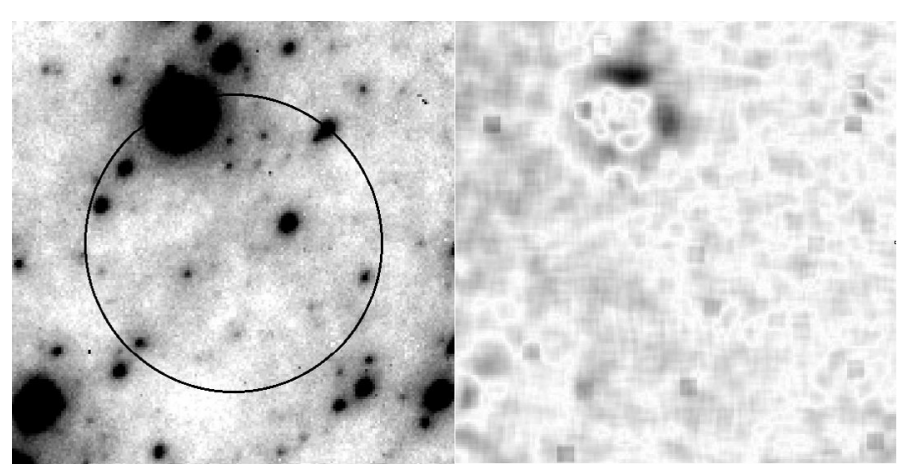

Fig. 3. Left panel: the field of Swift J1749.4-2807 observed in the $H$ band on 2010 Aug. 30. The black circle represents the X-ray error circle (1.6" radius, $90 \%$ c.1.). North is up and east to the left. Image size is $5.4^{\prime \prime} \times 5.4^{\prime \prime}$. Right panel: the result of image subtraction between the images obtained on Aug. 30 and Aug. 31.

NIR counterpart of Swift J1749.4-2807 has $H>23.5$ mag. A list of the candidate NIR counterparts of Swift J1749.4-2807 reporting their positions and $H$-band magnitudes, measured in the two observing epochs, is shown in Table 2.

\section{Discussion and conclusions}

An important goal of high-energy astrophysics is to derive or, at least, place tight constraints on the neutron star equation of state, probing the properties of ultradense matter. Astrophysics is the only branch of physics that can help since laboratory experiments cannot probe the relevant high-density regime (Lattimer \& Prakash 2006). As discussed in Sect. 1, the eclipsing AMXP Swift J1749.4-2807 would be among the best-suited objects known so far for dynamical studies aimed at mass measurements, because the radial velocity of the compact object $K_{1}$ and the system inclination $i$ can be measured with good precision. During quiescence, the optical/NIR flux of a low-mass X-ray transient is dominated by the companion star. The detection of the quiescent optical/NIR counterpart of Swift J1749.4-2807 
Table 2. Candidate NIR counterparts of Swift J1749.4-2807 (see Fig. 2).

\begin{tabular}{|c|c|c|c|}
\hline Candidate ID & $\begin{array}{c}\text { Position } \\
\text { RA(J2000), Dec(J2000) }\end{array}$ & $\begin{array}{l}H \text {-band magnitude } \\
\text { (Aug. 30) }\end{array}$ & $\begin{array}{l}H \text {-band magnitude } \\
\text { (Aug. 31) }\end{array}$ \\
\hline 1 & $17: 49: 31.88,-28: 08: 03.3$ & $16.26 \pm 0.05$ & $16.27 \pm 0.05$ \\
\hline 2 & $17: 49: 31.79,-28: 08: 04.5$ & $19.31 \pm 0.05$ & $19.37 \pm 0.05$ \\
\hline 3 & $17: 49: 31.75,-28: 08: 03.5$ & $19.55 \pm 0.05$ & $19.48 \pm 0.05$ \\
\hline 4 & $17: 49: 31.94,-28: 08: 04.3$ & $19.75 \pm 0.05$ & $19.66 \pm 0.05$ \\
\hline 5 & $17: 49: 31.92,-28: 08: 03.9$ & $19.85 \pm 0.05$ & $19.69 \pm 0.05$ \\
\hline 6 & $17: 49: 31.92,-28: 08: 06.1$ & $19.97 \pm 0.05$ & $19.88 \pm 0.05$ \\
\hline 7 & $17: 49: 31.91,-28: 08: 05.8$ & $20.65 \pm 0.05$ & $20.52 \pm 0.05$ \\
\hline 8 & $17: 49: 31.72,-28: 08: 05.1$ & $20.81 \pm 0.05$ & $20.74 \pm 0.05$ \\
\hline 9 & $17: 49: 31.87,-28: 08: 05.0$ & $21.07 \pm 0.06$ & $21.49 \pm 0.06$ \\
\hline $10(\mathrm{~A})$ & $17: 49: 31.84,-28: 08: 03.6$ & $21.09 \pm 0.08$ & $20.45 \pm 0.07$ \\
\hline 11 & $17: 49: 31.81,-28: 08: 03.5$ & $21.39 \pm 0.06$ & $21.10 \pm 0.06$ \\
\hline 12 & $17: 49: 31.83,-28: 08: 03.9$ & $21.41 \pm 0.06$ & $21.19 \pm 0.06$ \\
\hline 13 & $17: 49: 31.74,-28: 08: 06.1$ & $21.53 \pm 0.06$ & $21.89 \pm 0.07$ \\
\hline 14 & $17: 49: 31.95,-28: 08: 04.0$ & $21.65 \pm 0.07$ & $21.64 \pm 0.06$ \\
\hline 15 & $17: 49: 31.82,-28: 08: 03.0$ & $21.78 \pm 0.07$ & $21.84 \pm 0.07$ \\
\hline 16 & $17: 49: 31.96,-28: 08: 04.9$ & $21.79 \pm 0.15$ & $21.60 \pm 0.06$ \\
\hline 17 & $17: 49: 31.76,-28: 08: 03.6$ & $21.83 \pm 0.08$ & $22.06 \pm 0.07$ \\
\hline 18 & $17: 49: 31.81,-28: 08: 03.2$ & $21.99 \pm 0.08$ & $21.69 \pm 0.07$ \\
\hline 19 & $17: 49: 31.81,-28: 08: 03.9$ & $22.01 \pm 0.07$ & $21.59 \pm 0.06$ \\
\hline 20 & $17: 49: 31.83,-28: 08: 05.7$ & $22.05 \pm 0.07$ & $21.71 \pm 0.06$ \\
\hline $21(\mathrm{~B})$ & $17: 49: 31.80,-28: 08: 04.8$ & $22.11 \pm 0.07$ & $23.08 \pm 0.12$ \\
\hline 22 & $17: 49: 31.94,-28: 08: 05.0$ & $22.12 \pm 0.06$ & $22.28 \pm 0.07$ \\
\hline $23(\mathrm{C})$ & $17: 49: 31.85,-28: 08: 05.0$ & $22.12 \pm 0.08$ & $23.44 \pm 0.16$ \\
\hline 24 & $17: 49: 31.77,-28: 08: 05.4$ & $22.20 \pm 0.08$ & $21.94 \pm 0.12$ \\
\hline 25 & $17: 49: 31.72,-28: 08: 05.5$ & $22.22 \pm 0.07$ & $21.86 \pm 0.07$ \\
\hline 26 & $17: 49: 31.93,-28: 08: 05.9$ & $22.42 \pm 0.09$ & $22.09 \pm 0.07$ \\
\hline 27 & $17: 49: 31.73,-28: 08: 05.4$ & $22.54 \pm 0.08$ & $22.38 \pm 0.08$ \\
\hline 28 & $17: 49: 31.91,-28: 08: 04.3$ & $22.57 \pm 0.10$ & $23.00 \pm 0.12$ \\
\hline 29(D) & $17: 49: 31.79,-28: 08: 03.8$ & $22.57 \pm 0.10$ & $21.79 \pm 0.09$ \\
\hline 30 & $17: 49: 31.76,-28: 08: 05.6$ & $22.62 \pm 0.09$ & $23.14 \pm 0.14$ \\
\hline 31 & $17: 49: 31.86,-28: 08: 05.9$ & $22.66 \pm 0.10$ & $22.16 \pm 0.07$ \\
\hline 32 & $17: 49: 31.73,-28: 08: 04.5$ & $22.75 \pm 0.11$ & $22.66 \pm 0.09$ \\
\hline 33 & $17: 49: 31.81,-28: 08: 04.3$ & $22.81 \pm 0.11$ & $22.88 \pm 0.11$ \\
\hline 34 & $17: 49: 31.93,-28: 08: 05.3$ & $22.95 \pm 0.13$ & $22.61 \pm 0.09$ \\
\hline 35 & $17: 49: 31.78,-28: 08: 05.9$ & $23.28 \pm 0.14$ & $22.94 \pm 0.11$ \\
\hline 36 & $17: 49: 31.96,-28: 08: 04.7$ & $23.31 \pm 0.17$ & $23.43 \pm 0.20$ \\
\hline 37 & $17: 49: 31.83,-28: 08: 05.6$ & $23.47 \pm 0.22$ & $22.70 \pm 0.10$ \\
\hline 38 & $17: 49: 31.82,-28: 08: 06.4$ & $23.50 \pm 0.16$ & $23.68 \pm 0.20$ \\
\hline 39 & $17: 49: 31.82,-28: 08: 05.1$ & $23.52 \pm 0.24$ & $22.55 \pm 0.08$ \\
\hline 40 & $17: 49: 31.74,-28: 08: 04.4$ & $23.79 \pm 0.22$ & $23.43 \pm 0.20$ \\
\hline 41 & $17: 49: 31.76,-28: 08: 05.4$ & $23.99 \pm 0.35$ & $22.68 \pm 0.09$ \\
\hline
\end{tabular}

Notes. Magnitudes are in the Vega system and are not corrected for Galactic extinction. Errors are at $1 \sigma$ c.l.

would therefore make it possible to measure the companion star radial velocity $K_{2}$ providing the missing information to fully resolve the system and precisely determine its masses.

The companion star of Swift J1749.4-2807 can, in principle, be a young brown dwarf or a white dwarf, but both are too small to assure the filling of the Roche lobe, for the measured $8.82 \mathrm{~h}$ orbital period. The companion star is therefore a (possibly evolved) main sequence star. From geometrical considerations in an eclipsing system, Markwardt et al. (2010) and Altamirano et al. (2011) have derived a companion mass in the $0.46-0.81 M_{\odot}$ range for a neutron star with mass in the 0.8-2.2 $M_{\odot}$ range, and system inclinations in the $74.4^{\circ}-77.9^{\circ}$ range. Assuming an unirradiated main sequence companion star, these mass limits imply that the spectral type of the companion star should be in the K0V-M0V range. Independently of the compact object mass, the mean density $(\langle\rho\rangle)$ of a Roche lobe-filling companion star can be determined solely by the binary period: $\langle\rho\rangle=113 P_{\mathrm{h}}{ }^{-2} \mathrm{~g} \mathrm{~cm}^{-3}$, where $P_{\mathrm{h}}$ is the orbital period in hours (see, e.g., Faulkner et al. 1972). For Swift J1749.4-2807, with $P_{\mathrm{h}}=8.82$ h we obtain $M_{2}=0.8-1.0 M_{\odot}$ for the case of a main sequence companion, i.e. a G2V-K0V type star (Cox 2000). This makes the hypothesis of a main sequence companion star with spectral type later than $\mathrm{K}$ less favoured. Alternatively, a lower mass donor star can fill its Roche-lobe if it evolves within a Hubble time. According to this scenario, the system must have experienced the evolution of a more massive star before the onset of mass transfer, creating a common envelope phase with unstable mass transfer on a thermal time scale. The final product of this process is a low-mass $\mathrm{X}$-ray binary where a companion star has an evolved nucleus that transfers matter towards the compact object (see, e.g., Tauris \& van den Heuvel 2006). Using the evolutionary tracks of companion stars computed by Schenker \& King (2002), we obtain $M_{2} \sim 0.3-0.4 M_{\odot}$ for a system like Swift J1749.4-2807 in the limiting case of a donor star with a nearly all-helium nucleus. These considerations agrees with the $M_{2}$ estimates of Markwardt et al. (2010) and Altamirano et al. (2011) based on the system eclipses, with their lower and upper limits corresponding to an evolved or main sequence companion star, respectively.

In light of these considerations, knowing the source distance and the amount of interstellar absorption in its direction makes 
it possible to estimate the expected quiescent NIR luminosity of Swift J1749.4-2807. The detection of a type-I X-ray burst during the 2006 outburst provided an upper limit to the source distance of $6.7 \pm 1.3 \mathrm{kpc}$ (Wijnands et al. 2009). The measured $\mathrm{X}$-ray absorbing column density $\left(N_{H}=3.0 \times 10^{22} \mathrm{~cm}^{-2}\right.$; Ferrigno et al. 2011) is considerably higher than the total Galactic column density in the direction of the source $\left(N_{H}=1.1 \times 10^{22} \mathrm{~cm}^{-2}\right)$. This might indicate that a fraction of it is local to the system and possibly ionized (i.e. not contributing to the optical/NIR absorption). Assuming that the full observed column density contributes to the optical/NIR absorption, we estimate a colour excess $E(B-V)=5.41$ and $A_{H}=2.76 \mathrm{mag}$ (using the $N_{H} / E(B-V)$ conversion of Predehl \& Schmitt 1995 and the Galactic extinction curves of Fitzpatrick 1999) and would expect $H$-band apparent magnitudes in the range 20.4-21.3 for K0V and 21.7-22.6 for M0V main sequence stars (Cox 2000). These are conservative estimates that do not take into account that the companion star might be subjected to some irradiation from the compact object, even if the system is in quiescence. Furthermore, a lower effective absorption in the NIR band (or an evolved companion star) would result in brighter objects. The $3 \sigma$ upper limits of our two VLT observations are $H>23.5$ and $H>22.8$. Under these assumptions, we consider it a realistic possibility that we have actually detected the NIR counterpart of Swift J1749.42807 in our VLT-NACO images. As discussed in Sect. 2, the companion star should be searched primarily among the objects that show variability between the two consecutive nights of NACO observations. Assuming a variation of at least $0.1 \mathrm{mag}$ for the light curve of the Swift J1749.4-2807 companion star (as observed in other AMXPs, Shahbaz et al. 1998; Welsh et al. 2000; D'Avanzo et al. 2009 and references therein), we can exclude all objects with $H<21$ mag (Fig. 1). The remaining objects with $0.4<\Delta m<0.6$ are in principle the only valid candidates. Unfortunately, with just two epochs of observation we cannot distinguish between "real" variability and scatter due to the statistical dispersion of our photometry. We only point out that the four sources marked as "ABCD", with $H=21.1$, $H=22.1, H=22.1$, and $H=22.6$ mag, respectively, (Fig. 2 and Table 2) appear to be promising candidates, because their variability is greater than the observed dispersion for objects with similar magnitudes.

Finally, we note that the result of our image subtraction (Sect. 2) show a residual near the brightest star within the X-ray error circle (right hand panel of Fig. 2). If real, this would imply that the NIR quiescent counterpart of Swift J1749.4-2807 is "hidden" by a bright ( $H=16.3 \mathrm{mag}$ ) interloper (as observed for Aql X-1; Chevalier et al. 1999) making any future study during quiescence really challenging. However, the variability associated to such residual is not highly significant and can therefore be related to a spurious, incorrect subtraction of the bright star.

Future phase-resolved NIR observations (performed with large-aperture telescopes and adaptive optics) carried out covering a full orbital period would be fundamental for pinpointing the quiescent counterpart of Swift J1749.4-2807 through measuring its orbital-modulated variability, thereby opening the road for dynamical studies of this very promising source.
Acknowledgements. We thank the director of the European Southern Observatory for granting director's discretionary time (ID 285.D-5030(A)). P.D.A. and S.C. acknowledge tha Italian Space Agency for financial support through the project ASI I/009/10/0. The research leading to these results has received funding from the European Community's Seventh Framework Programme (FP7/2007-2013) under grant agreement number ITN 215212 "Black Hole Universe" and from the Spanish MEC under the Consolider-Ingenio 2010 Programme grant CSD2006-00070: First Science with the GTC (http:// www.iac.es/consolider-ingenio-gtc/).

\section{References}

Alard, C. 2000, A\&AS, 144, 363

Alard, C., \& Lupton, R. 1998, ApJ, 503, 325

Altamirano, D., Casella, P., Patruno, A., Wijnands, R., \& van der Klis, M. 2008, ApJ, 674, L45

Altamirano, D., Patruno, A., Heinke, C. O., et al. 2010, ApJ, 712, L58

Altamirano, D., Cavecchi, Y., Patruno, A., et al. 2011, ApJ, 727, L18

Blustin, A. J., Schady, P., \& Pandey, S. B. 2006, GCN Circ., 5207

Campana, S. 2009, ApJ, 699, 1144

Campana, S., Ravasio, M., Israel, G. L., Mangano, V., \& Belloni, T. 2003, ApJ, 594, L39

Casella, P., Altamirano, D., Patruno, A., Wijnands, R., \& van der Klis, M. 2008, ApJ, 674, L41

Chakrabarty, D., \& Morgan, E. H. 1998, Nature, 394, 346

Chakrabarty, D., Jonker, P. G., \& Markwardt, C. B. 2010, ATel, 2585

Chevalier, C., Ilovaisky, S. A., Leisy, P., \& Patat, F. 1999, A\&A, 347, L51

Cox, A. N. 2000, Allen's astrophysical quantities, 4th ed. (New York: AIP Press; Springer)

D’Avanzo, P., Campana, S., Casares, J., et al. 2009, A\&A, 508, 297

Falanga, M., Kuiper, L., Poutanen, J., et al. 2005, A\&A, 444, 15

Faulkner, J., Flannery, B. P., \& Warner, B. 1972, ApJ, 175, L79

Ferrigno, C., Bozzo, E., Falanga, M., et al. 2011, A\&A, 525, A48

Fitzpatrick, E. L. 1999, PASP, 111, 63

Galloway, D. K., Chakrabarty, D., Morgan, E. H., \& Remillard, R. A. 2002, ApJ, 576, L137

Galloway, D. K., Markwardt, C. B., Morgan, E. H., Chakrabarty, D., \& Strohmayer, T. E. 2005, ApJ, 622, L45

Hynes, R. 2010 [arXiv: 1010.5770]

Kaaret, P., Morgan, E. H., Vanderspek, R., \& Tomsick, J. A. 2006, ApJ, 638, 963 Khamitov, I., Bikmaev, I., Sakhibullin, N., et al. 2006, GCN Circ., 5205

Krimm, H. A., Markwardt, C. B., Deloye, C. J., et al. 2007, ApJ, 668, L147

Kubanek, P., Jelinek, M., \& French, J. 2006, GCN Circ., 5199

Lattimer, J. M., \& Prakash, M. 2006, NuPhA, 777, 479

Markwardt, C. B., \& Strohmayer, T. E. 2010, ApJ, 717, L149

Markwardt, C. B., Swank, J. H., Strohmayer, T. E., in't Zand, J. J. M., \& Marshall, F. E. 2002, ApJ, 575, L21

Melandri, A., Di Stefano, E., Covino, S., et al. 2006, GCN Circ., 5229

Papitto, A., Riggio, A., di Salvo, T., et al. 2010, MNRAS, 407, 2575

Papitto, A., Ferrigno, C., Bozzo, E., et al. 2011, ATel, 3556

Predehl, P., \& Schmitt, J. H. M. M. 1995, A\&A, 293, 889

Schenker, K., \& King, A. R. 2002, in The Physics of Cataclysmic Varibles and Related Objects, ed. B. T. Gansicke, K. Bevermann, \& K. Reinsch (San Francisco: ASP), ASP Conf., 261, 242

Shahbaz, T., Thorstensen, J. R., Charles, P. A., \& Sherman, N. D. 1998, MNRAS, 296, 1004

Steeghs, D., \& Casares, J. 2002, ApJ, 568, 273

Strohmayer, T. E., Markwardt, C. B., Swank, J. H., \& in’t Zand, J. 2003, ApJ, 596, L67

Tauris, T. M., \& van den Heuvel, E. P. J. 2006, in Compact Stellar X-Ray Sources, ed. W. H. G. Lewin, \& M. van der Klis (Cambridge University Press) Thorsett, S. E., \& Chakrabarty, D. 1999, ApJ, 512, 288

Yang, Y. J., Russell, R., Wijnands, R., et al. 2010, ATel, 2585

Welsh, W. F., Robinson, E. L., \& Young, P. 2000, AJ, 120, 943

Wijnands, R. 2006, in Trends in Pulsar Research, ed. J. A. Lowry (NY: Nova Science Pub. Inc.), 53

Wijnands, R., \& van der Klis, M. 1998, Nature, 394, 344

Wijnands, R., Rol, E., Cackett, E., et al. 2009, MNRAS, 393, 126 